Itinéraires Itinéraires

Littérature, textes, cultures

2019-1| 2019

La « renaissance littéraire » africaine en débat

\title{
Le visage de l'autre comme écotone dans Tout ce qu'on ne te dira pas, Mongo de Dany Laferrière
}

The Face of the Other as Ecotone in Tout ce qu'on ne te dira pas, Mongo

(Everything they won't tell you, Mongo) by Dany Laferrière

\section{Emmanuel Mbégane Ndour}

\section{OpenEdition}

\section{Journals}

Édition électronique

URL : http://journals.openedition.org/itineraires/6177

DOI : $10.4000 /$ itineraires.6177

ISSN : 2427-920X

Éditeur

Pléiade

Référence électronique

Emmanuel Mbégane Ndour, "Le visage de l'autre comme écotone dans Tout ce qu'on ne te dira pas, Mongo de Dany Laferrière », Itinéraires [En ligne], 2019-1 | 2019, mis en ligne le 24 juillet 2019, consulté le 01 août 2019. URL : http://journals.openedition.org/itineraires/6177 ; DOI : 10.4000/itineraires.6177

Ce document a été généré automatiquement le 1 août 2019

\section{$(\mathbb{P} \Theta \Theta$}

Itinéraires est mis à disposition selon les termes de la licence Creative Commons Attribution - Pas d'Utilisation Commerciale - Pas de Modification 4.0 International. 


\title{
Le visage de l'autre comme écotone dans Tout ce qu'on ne te dira pas, Mongo de Dany Laferrière
}

\author{
The Face of the Other as Ecotone in Tout ce qu'on ne te dira pas, Mongo
}

(Everything they won't tell you, Mongo) by Dany Laferrière

Emmanuel Mbégane Ndour

« Nous qui avec tant d'impatience rassemblons ces moi disjoints... acharnés à contenir la part inquiète de chaque

corps

dans cette obscurité difficile de nous. " Édouard Glissant, La Case du commandeur

\section{Introduction}

Le contexte d'« instabilité » politique et économique dans lequel nous vivons aujourd'hui à l'ère de la mondialisation est aussi marqué par la montée des nationalismes et des discours xénophobes dans la plupart des pays qui sont confrontés à l'Autre perçu comme problématique dans sa version la plus prosaïque et la plus dégradante, c'est-à-dire l'Autre vecteur de crises, d'instabilité et de transformation des identités nationales. C'est ainsi que le traitement politique de ce que l'on appelle (dans une ambiguité lexicale surprenante) la "crise des migrants ", " la crise des réfugiés » ou encore "la crise des sans-papiers ", se résume globalement à des politiques qui se réduisent de plus en plus au renforcement ou à la fermeture des frontières. Dans les territoires nationaux ${ }^{1}$ (l'emploi ici $\mathrm{du}$ mot n'est pas anodin: le territoire est souvent perçu par les nationaux comme un espace fermé devant être protégé dans son intégrité politique, sociale et culturelle alors qu'il peut aussi être un lieu fragmentaire, susceptible d'être déterritorialisé), la réaction générale se définit par une tension de plus en plus grande entre des élans de solidarité et d'accueil de l'Autre, d'une part, et la montée du racisme et de la xénophobie, d'autre part, 
qui se traduit souvent par la montée des partis d'extrême droite et par l'élection de personnages politiques au discours radical. La conclusion provisoire que l'on pourrait tirer de cette situation est que ce à quoi nous assistons aujourd'hui dans un monde de plus en plus globalisé, c'est à une fragmentation des espaces nationaux qui traduit une crise de l'universalisme, une incapacité à penser l'humanité comme un tout. La crise réelle dont il s'agit est une crise de la fraternité.

Dans ce contexte délétère, l'écrivain québécois d'origine haïtienne Dany Laferrière publie en 2015 un roman intitulé Tout ce qu'on ne te dira pas, $\mathrm{Mongo}^{2}$ pour participer au débat sur les valeurs du Québec initié par la femme politique québécoise Pauline Marois qui s'inspirait de la politique et du discours du président français de l'époque Nicolas Sarkozy. Tout ce qu'on ne te dira pas, Mongo raconte l'histoire d'un jeune immigré (Mongo) d'origine camerounaise qui rencontre, dans une rue de Montréal, le personnage de Dany Laferrière qui lui donne des conseils sur les codes de la société québécoise pour qu'il puisse s'approprier son nouveau lieu sans heurts. Le roman se présente donc du point de vue axiologique comme lieu de formation de valeurs, du point de vue esthétique (au sens de Jacques Rancière) comme une tentative de l'auteur de créer un espace partagé à travers la " construction d'un monde sensible commun » (Rancière $2000: 66$ ) et dans son écriture composite posée comme lieu paratopique qui figure le dédoublement entre l'auteur et l'univers de l'œuvre où convergent et se reconstruisent les identités individuelles (y compris l'éthos de l'auteur) et collectives.

Ma proposition est que le roman de Laferrière constitue dans sa narration et dans son écriture un écotone, un « lieu de rencontre entre deux communautés engagées dans un processus de créolisation et de germination pour former une nouvelle communauté » (Écotones 4, 2018), de nouvelles identités relationnelles. L'écotone est un espace de circulation qui peut également être perçu, selon Florence Krall, comme un théâtre de «conflits, de renouvellement entre plusieurs communautés» (Écotones 3, 2017). Cependant, ce qui se joue dans cet espace (tiers), c'est l'unidualité ${ }^{3}$ comme schéma de perception et de construction des relations sociales. L'enjeu de l'espace écotonique réside aussi dans l'hétéronomie, l'éthique de la responsabilité, la relation de transparence et d'opacité, la fluidité et l'instabilité des appartenances dans la redéfinition des structures d'imaginaires et des identités individuelles et collectives. Enfin, il s'agit d'un pari sur une écriture composite et paratopique qui met en scène la relation d'altérité par la figure dédoublée de l'auteur face à l'œuvre et le jeu de (re)construction des identités individuelles et collectives.

4 L'objectif de cet article est d'étudier le potentiel axiologique de la notion d'éthique du visage conçue comme écotone et d'explorer l'esthétique littéraire et linguistique à l'œuvre dans le texte de Laferrière. Cette esthétique est à envisager dans ses dimensions composite et paratopique en tant que lieu de refondation des identités individuelles et collectives.

5 Il s'agit donc dans un premier temps de voir comment Laferrière met en branle la question de la singularité du visage - du Soi et de l'Autre - dans le roman pour construire une "zone de contact», pour employer la formule de Mary Louise Pratt (1991), d'où sortiront des convergences de valeurs. Dans un deuxième temps, nous examinerons la manière dont s'articule la dialectique de l'Un et de l'Autre dans une écriture conçue comme tout ce qui peut "donner lieu à une inscription et à une interprétation " (Moulenda 2016: 11). Dans ce double mouvement, nous analyserons aussi le traitement, dans l'écriture du roman, des interrogations identitaires individuelles et collectives qui 
ont pour but de construire un lieu commun où s'élabore une nouvelle communauté composite.

\section{Pour une convergence axiologique}

6 L'esthétique de Laferrière dans Tout ce qu'on ne te dira pas, Mongo s'inscrit en faux par rapport à la conception et à la représentation dominante de l'altérité dans le monde occidental.

En Occident, toute approche de l'Autre passe par un appareil métaphysique qui, depuis Platon, enferme cette problématique dans un cercle vicieux : impossible de parler de l'Autre sans, en même temps, le restreindre dans les limites d'une représentation qui l'instrumentalise. Une représentation qui fait de lui en somme l'objet d'une manipulation. Les différentes philosophies qui sous-tendent la représentation de l'Autre dans l'ensemble des disciplines du savoir en Occident partagent un trait commun, celui d'assujettir son altérité au Même. (Durante 1997 :

3)

Aujourd'hui, ce constat d'opposition entre alter et ego dans de nombreux pays occidentaux est aggravé par la réduction du clivage entre des civilisations, des cultures et des populations non pas par une démarche d'ouverture et d'adhésion mais par une volonté d'assimilation de l'autre ou par une réaction de rejet.

7 Tout ce qu'on ne te dira pas, Mongo semble déployer une autre vision du monde qui s'appuie sur la philosophie de l'éthique du visage d'Emmanuel Levinas qui tente de sortir les relations interpersonnelles de la logique binaire du sujet d'un côté et de l'objet de l'autre. Pour Levinas, « [l]e visage est un geste d'expression qui est à la fois manière de se situer à l'égard du monde, et manière de parler à Autrui, de lui exprimer dans l'ouverture de la lumière l'intériorité de la conscience » (cité dans Misrahi 1999: 96). Ce qui sous-tend cette nouvelle relation, c'est la responsabilité que l'on a vis-à-vis de l'Autre comme fondement de notre subjectivité. L'approche du visage d'autrui se fait également chez Levinas dans une perspective non symétrique qui engage une responsabilité première. L'éthique du visage permet donc de construire le schéma d'une relation vertueuse dans des contextes d'immigration. L'intersubjectivité entre l'autre (le sujet migrant) et les populations des pays d'accueil se réalise ainsi dans l'unidualité, la fluidité du regard qui prend en charge la singularité et la complexité de l'autre, l'hétéronomie qui institue la responsabilité d'un rapport avec l'autre pour construire un espace commun. Ce rapport renouvelé s'établit dans une relation écotonique où l'altérité devient le tissu référentiel d'un monde partagé.

Dans le roman de Laferrière, le schéma de représentation de l'Un et de l'Autre se réalise dans la représentation du Québec et de Haïti dans la singularité de leur histoire. Pourtant la convocation de ces deux lieux aux trajectoires différentes dans une narration et dans un espace textuel communs (le roman de Laferrière, en l'occurrence), a aussi pour raison la volonté de mettre en évidence une convergence d'expériences quant à la situation coloniale du Québec et de Haïti et à la place de la langue française pour l'un et de l'anglais pour l'autre: «Je viens d'un pays où l'on s'est battu longtemps contre l'hégémonie de la langue française. Tout ce qu'on dit de l'anglais ici, je l'ai entendu là-bas à propos du français » (Mongo : 23). Les déictiques ici et là-bas structurent le schéma d'un écotone où l'Un et l'Autre s'inscrivent dans une communauté d'expériences et de destins face à la situation coloniale et postcoloniale. Cet écosystème nouveau prend les allures d'un paradigme non réductif selon lequel «autrui n'est ni un objet dans le champ de ma perception, ni un sujet qui me perçoit, c'est d'abord une structure du champ perceptif, 
sans laquelle ce champ dans son ensemble ne fonctionnerait pas comme il le fait" (Deleuze 1969 : 356-357). Ce champ perceptif situe le personnage de Laferrière dans un rapport de double appartenance au Québec et à Haïti mais aussi dans la symbolique d'« une manière d'être au monde nouvelle qui associe la mobilité et la multilocalité " (Agier 2018). Le nouveau visage du personnage de Laferrière se construit sur une éthique nouvelle. Il ne se pose ni comme sujet ni comme objet mais comme figure multiple, ouverte à la formation d'une nouvelle communauté.

D'autre part, la singularité de l'Un et de l'Autre se manifeste dans le roman à travers l'évocation de deux figures d'extériorité par rapport à Mongo : Pierre Eliot Trudeau et René Lévesque, deux hommes politiques marquants de l'histoire du Québec, (mais aussi) deux personnalités antagonistes sur le terrain politique : l'un symbolisant «le patricien arrogant » et l'autre "l'enfant chéri du peuple (Mongo: 82). Cette dualité s'estompe cependant dans une fraternité qui fait préséance, dont témoigne à nouveau Laferrière :

C'était fascinant, surtout quand les visages (de Trudeau et Lévesque) se superposaient pour ne faire qu'un [...] L'un, premier ministre du Québec ; l'autre, du Canada... Tous deux fils du Québec. [...] On ne peut pas trouver deux hommes aussi dissemblables, et d'une certaine manière aussi proches. (Mongo : 81-82)

Cette structure d'altérité domestique témoigne de la disposition de la société d'accueil, selon Laferrière, à jouer pleinement son rôle dans la construction de sa relation sociale avec le sujet migrant. C'est également pour Mongo une invitation à concevoir l'unidualité comme base d'une interrelation et d'une intersubjectivité nécessaires pour construire un espace commun avec le Québec. Une nouvelle éthique du visage qui repose sur l'unitédiversité du sujet lui est proposée comme modalité d'appropriation et d'appartenance au pays d'accueil.

10 Ce double schéma qui établit le face-à-face entre Mongo, Trudeau et Lévesque, d'une part et entre Trudeau et Lévesque, de l'autre, suggère l'établissement d'une relation sociale fondée sur l'hétéronomie de la part du sujet migrant. Celui-ci est invité à forger une perception de Soi et de l'Autre fondée sur la fluidité du regard pour construire un nouvel écotone. Laferrière situe la dualité de Trudeau et Lévesque dans une filiation biblique tout en déjouant la tentation d'une lecture binaire dans cette relation de fraternité paradoxale : «Certains ont vu une similitude avec la première scène d'affrontement de deux frères relatée dans la Bible : celle de Caïn et Abel. Le doux serait Lévesque et le cynique Trudeau. La réalité est, comme toujours, plus complexe » (Mongo: 83). Laferrière préconise donc de se situer aux intersections pourvoyeuses de perspectives multiples et de complexité. Le schéma antithétique que présente l'image des deux hommes politiques du Québec se résout dans une unidualité dont témoignent leurs tempéraments :

Lévesque par une certaine humanité. Trudeau, par une animalité certaine. Lévesque vous enveloppe. Trudeau vous étouffe. Mais les deux vous gardent dans leurs bras. Ils chassent différemment. Si Trudeau capture un cœur pour jouer (un serial killer), Lévesque préfère s'y installer à demeure (un serial lover). On aime Lévesque pour ses mains qui s'agitent comme des oiseaux autour de son visage quand il parle. On admire Trudeau pour ce côté sportif, insolent, moderne. (Mongo : 83)

La réconciliation de ces deux figures politiques du Québec suggère par la métaphore les tracés dans lesquels le sujet migrant - Mongo en l'occurrence - doit s'inscrire pour se forger une perception sans clivage et participer à la construction d'une nouvelle communauté. L'intention d'accueil et d'intégration se réalise ainsi dans l'invitation à la fluidité du champ perspectif deleuzien. La singularité du visage de l'Un et de l'Autre se définit non pas dans le schéma d'une opposition binaire entre «je » et l'« autre » mais par 
la reconstitution d'un espace de confluence et de remembrement de valeurs pour former une nouvelle communauté.

\section{Pour une grammaire commune}

11 Dans Tout ce qu'on ne te dira pas, Mongo, la construction d'un écotone se réalise également à travers l'écriture conçue elle-même comme un lieu de rencontre où le signe linguistique et le langage confluent pour l'avènement du sens. Dans le roman, celui-ci s'élabore d'abord par le paratexte conçu comme "seuils» du texte pour emprunter le terme de Genette $^{4}$, ou encore comme «mode d'emploi textuel ». Le titre du roman (Tout ce qu'on ne te dira pas, Mongo), constitue donc une porte d'entrée du roman qui introduit un schéma d'opacité et de transparence puisqu'il met en évidence l'existence de codes sociaux propres à toute collectivité et que les nouveaux venus ignorent. La société québécoise n'échappe pas à cette règle. Pour Laferrière, il s'agit de doter le sujet migrant d'outils d'intelligibilité de la société d'accueil. Cela se fait d'abord par une redéfinition nécessaire de l'exil et des situations qu'elle implique. Pour l'auteur :

L'exil, [...] c'est quitter l'île. L'ex-île. Partir sans pouvoir rien emporter avec soi. Peut-être ses coutumes, ses dieux, ses habitudes, son art de vivre, en somme, mais on découvre vite que tout cela ne vaut pas grand-chose ailleurs. Les autres ont aussi leurs coutumes auxquelles ils tiennent. (Mongo: 133)

Cette invitation à relativiser l'universalité de sa culture n'est pas une incitation à se replier sur soi-même, mais plutôt la formation par le langage d'un regard tourné vers le divers du monde. Il s'agit aussi de se déprendre de soi et d'une conception exclusiviste de sa culture, car «l'exil nous apprend que la perte, le manque et la distance sont au fondement même de toute appartenance, que le "chez-soi" est toujours provisionnel » (Zecchini 2010: 57). Ainsi advient un sens nouveau porté par un langage dont la dimension poétique est «la dimension à la fois créatrice et critique qui permet l'émergence de nouveaux mondes possibles » (Thomasset 2005 : 529). L'écotone provisoire survient dans l'expérience de soi et du monde à travers le langage de l'exil.

12 À la dé-construction sémantique du langage de l'« ex-île » succède une démarche didactique, car pour Laferrière :

l'exil est la plus grande école de conduite. On devrait envoyer tous les enfants faire un stage à l'école de l'exil. À ce jour, seuls les damnés de la terre semblent bénéficier de ce cours magistral. Dans cette obligation d'observer attentivement l'autre, on se découvre parfois. En analysant ainsi chacun de ses gestes, cela prend un temps avant de voir qu'on était en face d'un miroir. (Mongo : 77)

L'exil géographique mène à l'exil intérieur, car l'on redécouvre que s'ouvrir au monde de l'autre c'est aussi s'ouvrir à son propre monde, que l'exil nous ouvre à des voies hétérotopiques (au sens de Foucault) où l'on tente de réaliser l'impossible coïncidence ${ }^{5}$ avec soi-même. Il accrédite ainsi l'hétéronomie comme mode didactique dual pour la connaissance de l'homme et du monde. L'écriture de l'ex-île permet à Laferrière de construire un nouvel espace de rencontre dont le sens s'élabore dans la relation intriquée entre l'univers intime et le monde extérieur, entre l'intimité du soi et le visage de l'autre. L'écotone se dessine, dès lors, à travers cette double expérience du dedans et du dehors contre tout solipsisme de l'un et de l'autre.

Le partage d'expériences ou la transmission culturelle entre Laferrière et Mongo se fait ensuite par la mise en garde de ce dernier contre tout jugement prématuré : «Ne vous méprenez pas, il y a ici aussi des Montréalais curieux et passionnés. Ce sont des gens qui 
ne se dévoilent pas facilement » (Mongo: 8). L'affirmation de la complexité des membres de la société d'accueil est une manière d'établir un paradigme qui doit faire préséance aux interrelations entre les Montréalais et Mongo : la nécessité de prendre le temps de saisir l'autre dans sa singularité et dans sa complexité pour envisager ensuite un lieu de rencontre de la communauté reconstituée. L'auteur appelle à un remaniement des a priori de Mongo et ceux de tout sujet migrant pour leur substituer une patience et une prudence davantage propices à la con-naissance de l'autre. Dès lors, la complexité se pose dans le roman comme paradigme d'une éthique de l'autre dans la découverte de son visage authentique.

Par ailleurs, la formulation du titre du roman semble suggérer la possibilité d'en renverser le schéma unidirectionnel: Tout ce qu'on ne te dira pas, Mongo permet d'envisager, dans un parallélisme des formes, tout ce que Mongo ne dira pas (au Québec). Catherine (l'amie montréalaise de Mongo) décide de faire son mémoire de maîtrise sur Mongo parce que, dit-elle, c'est «toujours intéressant, un sujet vivant» (Mongo: 156), ignorant que « de l'autre en tant que tel, il n'y a peut-être rien à dire » (Mangeon 2008 : 73) selon la formule qu'Anthony Mangeon attribue à Bernard Mouralis. Malgré la maladresse de la formulation de Catherine, celle-ci constitue une tentative de relation pour une meilleure connaissance de l'autre. Le projet de cette dernière de faire de Mongo un sujet d'étude est, pour Laferrière, une provocation préventive pour éviter l'incongruité d'un amour teinté d'ethnologie. Pour l'auteur, il s'agit aussi de proposer le cadre d'un écotone où l'autre est perçu «comme subjectivité alternative ou comme subjectivation altérante » (Mangeon 2004 : 872), c'est-à-dire comme alter ego, porteur d'un imaginaire neuf. Ce qui se joue finalement dans ces interrelations, c'est aussi l'apprentissage et la découverte de la société d'accueil du sujet migrant pour que dans la relation de l'un et de l'autre se réalise l'écotone comme lieu de rencontre de la nouvelle communauté. C'est aussi dans ce cadre de réciprocité des échanges qui changent l'imaginaire de soi et de l'autre que peut se réaliser la refondation des identités individuelles et collectives.

La lecture paratextuelle du titre du roman permet également d'envisager Mongo comme l'alter ego de Laferrière confronté malgré l'ancienneté de son immigration à ce que la société québécoise n'a pas fini de lui dire. "Si je suis revenu sur mes pas, c'est pour te croiser en chemin " (Mongo: 158), dit-il à Mongo pour souligner que le chemin de l'immigration est une longue aventure faite d'apprentissage permanent, d'incessantes découvertes, de transparence, d'opacité et de rebours pour rencontrer l'altérité de son propre visage.

16 De plus, l'autre visage de Mongo pourrait être celui de l'écrivain camerounais que Laferrière pose également comme "subjectivité alternative». Mongo Beti dont l'identifiction de Laferrière sonne comme un hommage à ce que François Noudelmann appelle des airs de famille pour « la qualité de sa langue, son ton calme et réfléchi » (Mongo : 7) participe de ce que l'on pourrait appeler avec Patrick Chamoiseau «l'arbre relationnel " de Laferrière. François Noudelmann parle encore d'«affinités » et Chamoiseau (2013) des « fraternités littéraires » pour désigner le mouvement dans lequel se forment de nouvelles structures d'imaginaires, l'écotone témoin de la rencontre entre l'Afrique et les Antilles, Mongo Beti et Laferrière, pour dire l'avènement des nouvelles identités relationnelles fondées sur une éthique du visage.

17 Par ailleurs, cette démarche de com-préhension se réalise dans le cadre d'une éthique de la responsabilité. Les rapports interpersonnels se fondent également sur le désir de se 
connaître de l'un et de l'autre. Confronté à l'altérité de son propre visage sur une photo de jeunesse, Laferrière conjure «l'angoisse d'être vu» (Mongo : 132) par une démarche de responsabilité: ne pas se soustraire à l'expérience de l'autre mais plutôt saisir l'opportunité d'une relation féconde ${ }^{6}$. "Un visage qui ne connaît pas la lumière artificielle, qui n'a été vu que par des yeux nus de gens qui se plaçaient en face de moi. Il fallait s'approcher de moi si on voulait me regarder. Alors, j'ai pu aussi voir ceux qui m'ont vu » (Mongo : 132). C'est dans cette réciprocité que se réalise le miracle lévinassien de la présence de l'autre, dans l'épiphanie des hétéronomies multiples qui construisent l'espace écotonique à partager.

18 La couverture du roman de Dany Laferrière constitue également une voie d'accès à l'œuvre. Celle-ci montre le portrait de l'auteur le dos tourné, peut-être pour reprendre le chemin énigmatique du retour vers son passé d'immigré, d'où la rencontre avec Mongo. Puisque le paratexte se définit comme seuil du texte, l'image de Laferrière sur la page de couverture semble renvoyer au texte pour y découvrir l'autre visage de l'auteur : le visage inaccessible de l'image se dévoilant dans le texte. Ce schéma d'opacité et de transparence (Glissant) est repris dans une mise en abyme entre les notes pseudo-confidentielles du carnet noir du personnage de Laferrière et le récit destiné à Mongo et aux autres destinataires du texte romanesque. «Ce carnet abrite mes pensées secrètes, celles que je n'ai pas envie de balancer au visage des gens » (Mongo : 70), écrit Laferrière pour justifier cette disposition typographique qui opère la distribution du récit. Ce procédé d'enchâssement, marqué par le retrait typographique du texte, fait des destinataires des " pensées secrètes » de Laferrière, l'alter ego de Mongo, tout sujet migrant, tout lecteur du roman, les cofondateurs d'un nouvel écotone qui transgresse la frontière entre le réel et la fiction. Laferrière brise la «frontière mouvante mais sacrée entre deux mondes: celui où l'on raconte, celui que l'on raconte ", pour reprendre les mots de Genette cités par Greisch (2001: 180). Ce schéma métaleptique reconstitue un nouvel espace de rencontre qui situe l'expérience migratoire dans l'univers recomposé du réel et de la fiction. La lecture du paratexte de la page de couverture du roman suggère donc, dans l'esthétique de Laferrière, une invention ou une réinvention du réel ou la «reconstruction d'un monde sensible partagé » selon la formule de Rancière. L'effacement de la frontière entre l'extradiégétique et le diégétique dans Tout ce qu'on ne te dira pas, Mongo réalise la dynamique où doit se construire la nouvelle communauté : l'écotone où le visage de Mongo et celui du Québec reconstituent de nouvelles structures d'imaginaires.

\section{Une écriture (et) des identités composites}

19 Au-delà des seuils du texte, le texte lui-même. Dans Tout ce qu'on ne te dira pas, Mongo, celui-ci donne à voir ce qui dans l'écriture représente l'un et l'autre par la métaphore du schéma binaire que Laferrière présente dès le début du roman : "Voici l'état des choses au moment de mon arrivée à Montréal. À l'époque, le monde était à mes yeux composé de deux univers distincts : le Nord et le Sud. Haïti se trouvant au sud et le Québec au nord » ( Mongo : 13). Cette configuration géographique est relayée par une écriture composite faite d'une langue française propre à l'écrivain et de québécismes, tous deux reconfigurés dans une structure nouvelle qui rappelle le créole de Haïti. Un exemple de québécisme à propos de Catherine : "C'est qu'elle vient de tomber en amour ", écrit Laferrière (Mongo: 177). La formule « tomber en amour », traduction littérale de l'anglais (to fall in love) que 
l'on emploie dans le français québécois, se retrouve sous la plume de l'écrivain élu à l'Académie française qui définit sa langue comme de l'anglais mal traduit. C'est là qu'apparaît la dimension composite de cette langue aux racines multiples qui, en constituant un lieu de rencontre, dit ce qu'elle fait: dessiner l'écotone textuel d'une nouvelle communauté composée d'origines diverses : d'Africains, d'Haïtiens, de Québécois et de bien d'autres.

L'écriture de Laferrière est une écriture qui établit des «zones de contact ", là où se reforment les identités individuelles et collectives. Celles-ci se constituent dans l'ailleurs : le Québec pour Mongo, la Floride pour Laferrière, lieux écotoniques où le visage de l'autre se révèle être l'altérité de son propre visage. « Ma vie est cousue de là-bas. J'ai toujours voulu aller là-bas pour voir si j'y étais. Et, bien sûr, il m'arrive de me croiser dans un café en train de lire une nouvelle de Borges. C'est ainsi que j'ai su que c'était moi » (Mongo: 134-135). Cette altérité du même situé dans un autre lieu dédouble la " non-coïncidence » (Sartre) de soi à soi chez Laferrière et renforce l'acuité du regard sur lui-même. Ce déplacement géographique est également un déplacement temporel: Laferrière, l'écrivain d'aujourd'hui, c'est Mongo l'immigré d'hier. L'on entre ainsi dans le schéma de la récursivité des identités qui miment le tracé des écotones d'où doivent s'établir les contacts entre l'altérité de son propre visage et celle du visage de l'autre.

21 L'écriture du roman est encore un " être singulier qui (s')écrit » selon Gallinari. Instance paratopique, « cette créature para-graphique est située dans une temporalité particulière établie par des exigences scripturales » (Gallinari 2009: 4). Celle-ci est intrinsèque à l'œuvre qui oscille d'un côté entre l'histoire du Québec et celle de Laferrière dans son expérience migratoire et de l'autre, la période contemporaine où la sociologie du Québec change de visage avec l'arrivée de migrants. L'écriture elle, tente de réaliser un autre impossible : celui de définir la place de l'auteur dans son champ discursif et dans la société.

Elle est dès lors caractérisée [...] par des «petits moments » existentiels consacrés au «faire artistique» et marqués par des activités de création, telles que (indépendamment des époques) prendre des notes dans les espaces de relation sociale, effectuer des recherches, lancer des discussions, oser des expérimentations, observer des comportements, inscrire les traces des êtres et des choses, enregistrer des rêves et fantaisies, penser l'œuvre, etc. - ou, simplement, écrire en fonction des paramètres esthétiques-culturels caractéristiques de moments historiques diversifiés. (Gallinari 2009 : 4-5)

Ces activités (la sortie au café où Laferrière prend des notes dans son carnet noir, où Mongo le retrouve pour discuter avec lui; les chroniques à la radio pour parler de questions liées à l'actualité québécoise et mondiale) constituent la réalisation d'une écriture paratopique dans une période (d'incertitude voire d'inquiétude) où la question migratoire occupe toutes les instances sociopolitiques. Surtout, elle advient à l'instigation d'un auteur (Laferrière) soucieux de prendre part au débat actuel sur l'immigration au Québec mais aussi de «construire le territoire de son œuvre» (Maingueneau 2004: 85) par un discours constituant à travers lequel il redessine les multiples facettes de son identité. L'écriture devient ainsi l'écotone où confluent l'auteur et l'univers de son œuvre, dans une relation paradoxale où celui-ci redécouvre à travers elle l'altérité de son propre visage et tente de reconstruire les différentes facettes de son identité et celle de la société. Ainsi, Laferrière s'exprime dans ces termes :

Je crois avoir fait un petit livre assez spontané où j'ai laissé mes expériences remonter tranquillement à la surface. J'ai noté chaque idée ou image qui m'a traversé l'esprit. Juste des notes qui pourront aider, j'espère, un jeune homme qui vient d'arriver à Montréal. Les gens qui n'ont jamais quitté leur pays ne peuvent 
savoir ce qu'il coûte de se retrouver dans un jeu dont on ignore les règles. (Mongo :

191)

$\mathrm{Au}$ « faire artistique » s'adjoint la volonté pour l'auteur de construire « le territoire de son œuvre " à travers une anamnèse tranquille. Son écriture prend en charge à la fois les possibles préoccupations de tout sujet migrant - aussi celle de Mongo, son alter ego mais s'attache également à informer la société d'accueil sur les difficultés et les douleurs de l'exil. Ainsi se forme l'écotone dans une triangulation paratopique (Laferrière, son œuvre et la société) où s'élaborent les nouvelles identités individuelles et collectives. À la question de savoir pourquoi il a écrit le roman, Laferrière répond : " pour tenter de mieux connaître ce monde agité qui m'entoure, mais surtout que ça me donne la possibilité d'écrire » (Mongo: 191). Ainsi se construit l'ethos de l'écrivain à travers « l'ethos de la littérature comme appréhension, arpentage et lecture différenciée du monde » (BerrouëtOriol 2013).

L'autre visage de lui-même se révèle aussi dans son identité d'écrivain. Celle-ci n'est pas révélée a priori ; son accès se fait de manière non immédiate. Pour marquer ce schéma de transparence et d'opacité, Laferrière situe l'accès au visage de l'écrivain dans les confidences du carnet noir :

La vie d'un écrivain se résume à ce mélange d'encre et de sang. Un jour, c'est un peu de sang dans beaucoup d'encre, et le jour suivant c'est un peu d'encre dans beaucoup de sang. Un sang d'encre. Donc un seul conseil, si jamais tu ne te dérobes pas face à ce destin d'écrivain que je pressens : n'écoute pas les sirènes du passé qui te promettent souvent un confort identitaire. Cherche plutôt à te mettre en danger en prenant un chemin inédit. (Mongo: 158)

Le visage de l'un et de l'autre se retrouvent dans la métaphore du sang d'encre, dans l'unidualité du sujet migrant dont l'identité prend les chemins inédits des écotones aux apports composites. Le visage de l'un et de l'autre, celui de Mongo et de Laferrière se réalisent dans une hétéronomie qui est fonction d'un champ perceptif nouveau : lieu de rencontre et de germination pour que les questions migratoires se résorbent dans des fraternités relationnelles.

\section{Conclusion}

Tout ce qu'on ne te dira pas, Mongo propose une manière d'appréhender ce qui semble être la question majeure de ce début de $\mathrm{xxI}^{\mathrm{e}}$ siècle : la question migratoire et ce qu'elle révèle de «notre impossibilité à envisager l'unité-diversité, les solidarités conflictuelles, les ruptures qui rassemblent, les écarts convergents : une complexité que seule l'idée d'une mise en Relation peut nous aider à fréquenter » (Chamoiseau 2013 : 15).

Le roman, par le biais de la double appartenance, déploie une structure du champ perceptif susceptible de révéler la possibilité de construire un espace de convergence d'identités. Par la convocation de personnages représentant des personnalités politiques québécoises, Laferrière pose l'unidualité et l'hétéronomie comme fondements d'une éthique nouvelle et d'une subjectivité nécessaire pour construire une nouvelle communauté.

Par ailleurs, l'écriture du roman pose le paratexte comme seuil du texte permettant des inférences lectoriales du titre et de la page de couverture du roman pour doter le sujet migrant - Mongo - et le Québec, sa société d'accueil, d'outils de compréhension mutuelle et de construction d'un lieu commun. Ces outils qui ont pour nom: relativisme, subjectivités alternatives, responsabilité et transgression de la frontière entre le réel et la 
fiction, s'avèrent nécessaires à l'édification d'une éthique du visage permettant la rencontre avec soi et avec l'autre. Celle-ci est également orchestrée au cœur d'un lieu paratopique - l'écriture - où confluent l'auteur et son univers romanesque pour reformuler les identités multiples de l'écrivain et de la société qu'il évoque.

Enfin, Tout ce qu'on ne te dira pas, Mongo mobilise une langue composite qui établit des «zones de contact» entre le visage de l'un et de l'autre, celui de Mongo et celui de Laferrière, celui de l'homme et celui de l'écrivain dans un schéma de transparence et d'opacité. La récursivité des identités procède d'un champ perspectif nouveau qui suggère l'édification d'un lieu commun communautaire. Il convient de citer Laferrière une dernière fois : « La littérature est là pour dire que nous ressentons les choses de la même manière quels que soient les climats, les paysages, les classes, les races, les sexes et les religions. L'émotion pure est interchangeable et unique malgré tout » (Mongo: 249). C'est dans ce propos que le roman réalise l'ultime tentative de construire un espace commun fait d'apports composites, le visage de l'un et de l'autre : l'écotone où se constituent les nouvelles identités relationnelles.

\section{BIBLIOGRAPHIE}

Agier, Michel, 2018, « Regarder le monde comme il fait monde à partir de toutes les situations d'entre-deux », Entretien de Anne Bocandé avec Michel Agier, Revue Africultures, [En ligne], http://africultures.com/regarder-monde-monde-a-partir-de-toutes-situations-dentre-deux/, consulté le 23 juillet 2019.

Bakhtine, Mikhaill, 1970, Problèmes de la poétique de Dostoïevski, Paris, Seuil.

Berrouët-Oriol, Robert, 2013, « Notre Québec à tous », Lettre ouverte à Andrée Ferretti, publiée à Montréal le 14 décembre 2013, [En ligne], http://berrouet-oriol.com/droit-de-parole/ notrequebecanoustous/, consulté le 23 juillet 2019.

Chamoiseau, Patrick, 2013, Césaire, Perse, Glissant. Les liaisons magnétiques, Paris, Éditions Philippe Rey.

Deleuze, Gilles, 1969, « Michel Tournier et le monde sans autrui », dans Logique du Sens, Paris, Minuit, p. 354-357.

Durante, Daniel Castillo, 1997, « Les enjeux de l'altérité et la littérature », dans F. Tétu de Labsade (dir.), Littérature et dialogue interculturel, Québec, Les Presses Universitaires de Laval.

«ÉCOTONES 3 : océan indien - écotones, zones de contact et tiers-espaces », Appel à contribution, Calenda, Publié le jeudi 28 septembre 2017, https://calenda.org/416478, consulté le 23 juillet 2019 .

«ÉCOTONES 4 : Partitions and Borders », Appel à contribution, Université de Pennsylvanie, publié le 4 janvier 2018, [En ligne], https://call-for-papers.sas.upenn.edu/cfp/2018/01/04/ ecotones-4-kolkata, consulté le 23 juillet 2019.

Gallinari, Melliandro Mendes, 2009, «La "clause auteur" : l'écrivain, l'ethos et le discours littéraire ", Argumentation et Analyse du Discours, n 3, [En ligne], https:// 
journals.openedition.org/aad/663, consulté le 23 juillet 2019.

DOI : $10.4000 /$ aad.663

Genette, Gérard, 1987, Seuils, Paris, Seuil.

Greisch, Jean, 2001, Paul Ricœur. L'itinéraire du sens, Grenoble, Éditions Jérôme Millon.

Laferrière, Dany, 2015, Tout ce qu'on ne te dira pas, Mongo, Montréal, Mémoire d'encrier.

Levinas, Emmanuel, 1982, Éthique et Infini, Paris, Fayard.

Maingueneau, Dominique, 1996, Les Termes clés de l'analyse du discours, Paris, Seuil.

Maingueneau, Dominique, 2004, Le Discours littéraire, paratopie et scène d'énonciation, Paris, Armand Colin.

Mangeon, Anthony, 2008, « Avec Bernard Mouralis », Études littéraires africaines, n² 26, p. 73-75,

[En ligne], https://id.erudit.org/iderudit/1035126ar, consulté le 23 juillet 2019.

DOI : 10.7202/1035126ar

Mangeon, Anthony, 2004, Lumières noires, discours marron. Indiscipline et transformations du savoir chez les écrivains noirs américains et africains; itinéraires croisés d'Alain Leroy Locke, V. Y. Mudimbe et de leurs contemporains, Thèse de doctorat, Université de Cergy-Pontoise.

Misrahi, Robert, 1999, Qui est l'autre ?, Paris, Armand Colin.

Moulenda, Joseph Igor, 2016, « Par-delà le sens, l'écriture et le texte. Jacques Derrida », Controverses. Revue spécialisée de philosophie, ENS, Libreville, Éditions Oudjat, [En ligne], hal-01504272.

Noudelmann, François, 2012, Les Airs de famille. Une philosophie des affinités, Paris, Gallimard.

Pratt, Mary Louise, 1991, « Arts of the Contact Zone », Profession, p. 33-40, [En ligne], http:// www.jstor.org/stable/25595469, consulté le 23 juillet 2019.

Rancière, Jacques, 2000, Le Partage du sensible. Esthétique et politique, Paris, La Fabrique-éditions.

Sartre, Jean-Paul, 1943, L'Être et le Néant, Paris, Gallimard.

Sartre, Jean-Paul, 1948, "Orphée noir », dans Anthologie de la nouvelle poésie nègre et malgache de langue française, Paris, PUF.

Thomasset, Alain, 2005, «L'imagination dans la pensée de Paul Ricœur : fonction poétique du langage et transformation du sujet ", Études théologiques et religieuses, vol. 80, n ${ }^{\circ}$ 4, p. 525-541, [En ligne], https://www.cairn.info/revue-etudes-theologiques-et-religieuses-2005-4-page-525.htm, consulté le 23 juillet 2019 .

DOI : $10.3917 /$ etr.0804.0525

Wulf, Christoph, 2008, « Homo absconditus. L'anthropologie fondamentale d'Edgar Morin », Synergies Monde, $\mathrm{n}^{\circ}$ 4, p. 263-266.

Zecchini, Laetitia, 2010, «"Je suis le multiple”. Exil historique et métaphorique dans la pensée d'Edward Said », Tumultes, n 35, p. 49-65, [En ligne], https://www.cairn.info/revuetumultes-2010-2-page-49.htm, consulté le 23 juillet 2019.

DOI : $10.3917 /$ tumu.035.0049 


\section{NOTES}

1. L'emploi ici du mot n'est pas anodin : le territoire est souvent perçu par les nationaux comme un espace fermé devant être protégé dans son intégrité politique, sociale et culturelle alors qu'il peut aussi être un lieu fragmentaire, susceptible d'être déterritorialisé.

2. La forme abrégée « Mongo » sera désormais utilisée pour les mentions du titre.

3. Dans ses travaux, Edgar Morin attribue à l'homme une dimension ambivalente qui en fait un «être à la fois biologique, naturel et cérébral d'une part, culturel, social et spirituel de l'autre, l'un étant inséparable de l'autre » (cité dans Wulf 2008 : 264).

4. Dominique Maingueneau, citant Gérard Genette précise que le paratexte est destiné à «rendre présent le texte, pour assurer sa présence au monde, sa "réception" et sa consommation » $(1996: 60)$.

5. Selon Mikhaïl Bakhtine, « L'homme ne coïncide jamais avec lui-même » (1970:97).

6. Jean-Paul Sartre, dans "Orphée noir ", souhaitait à ses contemporains « de ressentir comme [lui] le saisissement d'être vus » (Sartre 1948).

\section{RÉSUMÉS}

Cet article élabore une réflexion sur le visage de l'autre conçu comme écotone dans le roman de Laferrière, à savoir un lieu où la singularité de l'un et de l'autre se rencontrent pour construire une autre vision du monde qui repose sur des valeurs convergentes. L'éthique du visage d'Emmanuel Levinas permet de mener cette réflexion qui interroge également une écriture composite et paratopique à envisager comme lieu d'inscription et d'interprétation des signes écotoniques dans l'œuvre, d'interrogation et de (re)formation des identités individuelles et collectives et d'élaboration d'une nouvelle communauté composite.

This article analyses the conception of the face of the other in Laferrière's novel as an ecotone, that is, a place where two individuals meet in order to build a renewed vision of the world based on their converging values. This analysis is informed by Emmanuel Levinas' ethics of the face. It considers composite and paratopic writing as a place where ecotonic signs in the novel are inscribed and may be interpreted. These signs serve to question and (re)-shape individual and collective identities and to create a new composite community.

\section{INDEX}

Keywords : face, the other, Laferrière (Dany), Levinas (Emmanuel), ecotone, migration, exile, identity

Mots-clés : visage, l'autre, Laferrière (Dany), Levinas (Emmanuel), écotone, migration, exil, identité 
AUTEUR

EMMANUEL MBÉGANE NDOUR

University of the Witwatersrand 